\title{
Predictors of residual pulmonary vascular obstruction after anticoagulation monotherapy in patients with intermediate-risk pulmonary embolism
}

\author{
Youjin Chang $^{1 \# \wedge}$, Jae Young Moon ${ }^{2 \#}$, Jae-Hyeong Park ${ }^{3} \wedge$, Sangbong Choi ${ }^{1 \wedge}$, Hyuk Pyo Lee ${ }^{1 \wedge}$, \\ Jae Kyeom Sim ${ }^{4} \wedge$, Young Seok Lee ${ }^{4} \wedge$
}

${ }^{1}$ Division of Pulmonary and Critical Care Medicine, Department of Internal Medicine, Sanggye Paik Hospital, Inje University College of Medicine, Seoul, Korea; ${ }^{2}$ Division of Pulmonology and Critical Care Medicine, Department of Internal Medicine, Chungnam National University Sejong Hospital, Chungnam National University College of Medicine, Daejeon, Korea; ${ }^{3}$ Department of Cardiology in Internal Medicine, Chungnam National University Hospital, Chungnam National University School of Medicine, Daejeon, Korea; ${ }^{4}$ Division of Pulmonary, Allergy, and Critical Care Medicine, Department of Internal Medicine, Korea University Guro Hospital, Seoul, Korea

Contributions: (I) Conception and design: Chang Y, Moon JY, Lee YS; (II) Administrative support: Chang Y, Moon JY, Lee YS; (III) Provision of study materials or patients: All authors; (IV) Collection and assembly of data: All authors; (V) Data analysis and interpretation: All authors; (VI) Manuscript writing: All authors; (7) Final approval of manuscript: All authors.

\#These authors contributed equally to this work.

Correspondence to: Young Seok Lee, MD, PhD. Division of Pulmonary, Allergy, and Critical Care Medicine, Department of Internal Medicine, Korea University Guro Hospital, 148, Gurodong-ro, Guro-gu, Seoul 08308, Korea. Email: avonlea76@korea.ac.kr.

Background: Patients with intermediate-risk pulmonary embolism (PE) can be treated with anticoagulation monotherapy. However, clinicians are concerned as to whether anticoagulation monotherapy is sufficient to reduce mortality in patients with a large embolic burden, and to resolve vascular obstruction. We investigated whether anticoagulation monotherapy was appropriate in patients with intermediate risk $\mathrm{PE}$ in terms of the occurrence of residual pulmonary vascular obstruction (RPVO), and the factors that independently predict the occurrence of RPVO.

Methods: This was a multicenter retrospective observational study of patients at intermediate risk of PE who were admitted to three hospitals between January 2012 and December 2017.

Results: Of total 91 patients, the median age was 72 years and 37 (40.7\%) were male. Twenty-five patients (27.5\%) were diagnosed with RPVO during follow-up. Multivariate logistic regression revealed chronic lung disease [odds ratio (OR), 4.14; 95\% confidence interval (CI), 1.243-13.797; $\mathrm{P}=0.021$ ] and the ratio of the diameters of the main pulmonary artery and ascending aorta ratio (P/A ratio) $>1.0$ documented on a chest computed tomography (CT) at presentation (OR, 3.46; 95\% CI, 1.113-10.770; $\mathrm{P}=0.032$ ) were significant independent predictors of RPVO occurrence. The incidence of RPVO in patients without these two factors was only $9.7 \%$, but in those with the two factors it was $60 \%(\mathrm{P}=0.004)$.

Conclusions: Anticoagulation monotherapy did not seem to be a sufficient treatment to reduce RPVO, but the outcome was similar to that of patients treated with other therapies. Therefore, considering the riskbenefit ratio, we do not need to change the initial treatment as systemic thrombolytic therapy or catheterbased therapy in patient with intermediate risk PE. Underlying chronic lung disease and a P/A ratio $>1$ on the initial chest CT predicted the occurrence of RPVO. Therefore, we should carefully assess persistent of dyspnea and exercise limitations using various methods in patients with these risk factors, to detect the occurrence of chronic thromboembolic pulmonary disease (CTEPD) earlier.

\footnotetext{
^ ORCID: Youjin Chang, 0000-0002-4838-466X; Jae Young Moon, 0000-0001-8724-6289; Jae-Hyeong Park, 0000-0001-7035-286X; Sangbong Choi, 0000-0003-0532-3557; Hyuk Pyo Lee, 0000-0002-6712-5647; Jae Kyeom Sim, 0000-0003-0873-2807; Young Seok Lee, 0000-0002-0144-2033.
} 


\begin{abstract}
Keywords: Pulmonary embolism (PE); residual pulmonary vascular obstruction (RPVO); anticoagulants; quality
\end{abstract} of life; chronic thromboembolic pulmonary disease (CTEPD)

Submitted Mar 09, 2021. Accepted for publication May 31, 2021.

doi: $10.21037 /$ jtd-21-403

View this article at: https://dx.doi.org/10.21037/jtd-21-403

\section{Introduction}

Pulmonary embolism (PE) occurs frequently in hospitalized patients. Treatment for PE is based on risk stratification for mortality (1-7). Patients with PE are classified as being at low, intermediate, or high risk of mortality, based on vital signs [e.g., blood pressure (BP)] and right ventricle (RV) pressure overload (e.g., RV dysfunction and/or elevated cardiac enzymes) (1-7). In general, low-risk patients are treated with anticoagulation monotherapy, and high-risk patients are treated with systemic thrombolysis or surgical embolectomy $(1,2)$. In patients with intermediate risk PE, anticoagulation monotherapy was shown to be effective in reducing mortality and is therefore recommended by current guidelines $(1,2)$. However, clinicians are still concerned as to whether anticoagulation monotherapy is sufficient to reduce mortality in patients with a large embolic burden, and to resolve vascular obstruction (8-11).

According to previous studies, many patients treated with PE show residual pulmonary vascular obstruction (RPVO) on follow-up imaging after terminating PE therapy (12-18). Because RPVO is associated with the recurrence of PE and occurrence of chronic thromboembolic pulmonary disease (CTEPD), with or without pulmonary hypertension (PH), patients with RPVO have a lower quality of life than those without RPVO $(12-17,19,20)$. In patients with RPVO, persistent dyspnea and exercise limitations due to dyspnea are important symptoms because they may indicate CTEPD or CTEPH. In addition, treating CTEPD and CTEPH improves quality of life (14,21-23). Thus, current guidelines recommend that clinicians confirm the presence of RPVO via follow-up imaging in patients with persistent dyspnea and exercise limitations after terminating PE therapy $(2,14)$. We hypothesized that anticoagulation monotherapy does not reduce the occurrence of RPVO due to the large embolic burden in patients with intermediate risk PE, and that identifying the factors that predict the occurrence of RPVO in these patients would allow earlier detection of CTEPD and CTEPH (because the clinician is more likely to detect these diseases in patients with risk factors for
RPVO). Thus, we investigated whether anticoagulation monotherapy was appropriate in patients with intermediate risk $\mathrm{PE}$ in terms of the occurrence of RPVO, and the factors that independently predict the occurrence of RPVO.

We present the following article in accordance with the STROBE reporting checklist (available at https://dx.doi. org/10.21037/jtd-21-403).

\section{Methods}

\section{Study overview}

This was a multicenter retrospective observational study of patients with intermediate risk PE who were admitted to three university-affiliated hospitals in South Korea between January 2012 and December 2017. A subset of these patients was selected by applying inclusion and exclusion criteria. All patients were diagnosed by echocardiography, chest computed tomography (CT), and cardiac markers and treated with anticoagulation monotherapy, in accordance with international guidelines $(1,2)$. Anticoagulation monotherapy included initial heparin treatment [e.g., conventional heparin or low-molecular weight heparin (LMWH) for 5 days, followed by warfarin or non-vitamin $\mathrm{K}$ antagonist oral anticoagulant (NOAC) for 3-6 months] and initial NOAC treatment (e.g., NOAC use from first day at admission to 3-6 months). In patients using conventional heparin, we intravenously injected conventional heparin and titrated the dose to maintain an activated partial thromboplastin time of $60-80 \mathrm{sec}$. In patients using $\mathrm{LMWH}$, we subcutaneously injected $1 \mathrm{mg} / \mathrm{kg}$ of LMWH every 12 hours. In patients using oral administration of warfarin or NOAC, the dose of warfarin was titrated to maintain an international normalized ratio of 2-2.5 and the NOAC dose was determined based on the manufacturer's recommendations $(1,2)$. The primary outcomes were the incidence of RPVO and factors predicting the occurrence of RPVO after anticoagulation monotherapy in patients with intermediate risk PE.

This study was conducted in accordance with the 
Declaration of Helsinki (as revised in 2013). This study was approved by the Institutional Review Boards (IRBs) of all participating hospitals and the Korea Medical Center IRB (approval No.: 2020GR0057). We ensured protection of patient privacy and anonymity. The need for informed consent was waived because of the retrospective nature of the study.

\section{Patients}

The inclusion criteria were patients aged above 20 years diagnosed with an intermediate risk PE; these patients were treated with anticoagulation monotherapy. In addition, all included patients had follow-up images (e.g., chest CT or echocardiography) after 3-6 months of PE therapy. The exclusion criteria were as follows: patients with acute PE who were at low or high risk of mortality; patients initially treated with anticoagulation therapy combined with thrombolytic therapy (e.g., systemic thrombolysis or catheter-based therapy); patients without a follow-up chest CT or follow-up echocardiography after 3-6 months of PE treatment; and patients with insufficient electronic medical records.

\section{Definition}

Acute PE was defined as the occurrence or aggravation of dyspnea within 15 days before the diagnosis, with an embolism in the pulmonary artery confirmed by chest CT $(1,2)$. Patients with intermediate risk PE were those with an acute PE without hemodynamic instability, along with RV dysfunction confirmed by echocardiography, or myocardial injury confirmed by elevated cardiac markers, including troponin $\mathrm{I}>0.06 \mu \mathrm{g} / \mathrm{L}$ or troponin $\mathrm{T}>0.01 \mu \mathrm{g} / \mathrm{L})(8)$. Hemodynamic instability was defined as the need for cardiopulmonary resuscitation, systolic $\mathrm{BP}<90 \mathrm{mmHg}$ with evidence of end-organ hypoperfusion, and vasopressors required to achieve $\mathrm{BP}>90 \mathrm{mmHg}$ despite adequate filling status with evidence of end-organ hypoperfusion (2). RV dysfunction on echocardiography was defined as an enlarged $\mathrm{RV}$ and flattened interventricular septum in the parasternal long axis view, dilated $\mathrm{RV}$ with a basal $\mathrm{RV}$ diameter/left ventricle diameter ratio (RV/LV ratio) $>1.0$ and McConnell sign on the four-chambered view, and decreased tricuspid annular plane systolic excursion measured in M-mode (<16 mm) (2). RPVO was defined as persistent pulmonary vascular obstruction according to imaging studies regardless of symptoms (e.g., dyspnea or exercise limitations) after
3-6 months of anticoagulation monotherapy based on international guidelines $(13,16-18)$. Comorbidities were those previously diagnosed by a physician and based on the traditional indicators. Chronic lung disease included chronic obstructive lung disease, interstitial lung disease, tuberculosis destroyed lung, and bronchiectasis. Chronic liver disease included chronic hepatitis and liver cirrhosis. Recent surgery was defined as surgery performed within 3 months before diagnosis. Immobilization was defined as immobility due to various causes for over 3 days within 1 month before the PE diagnosis. Estrogen use was defined as estrogen intake during the 1 month before the diagnosis of PE. Central lesions at the PE location were defined as the presence of a saddle-shaped thrombus in the main pulmonary artery or the presence of a thrombus in the left or right main pulmonary artery. Peripheral lesions were defined as the presence of a thrombus not in the main pulmonary artery but rather in the lobar, segmental, or subsegmental pulmonary arteries. The ratio of the diameters of the main pulmonary artery and ascending aorta ( $\mathrm{P} / \mathrm{A}$ ratio) was determined at the level of the bifurcation of the pulmonary artery as seen on the chest CT image. Hospital mortality was defined as death due to PE between admission and discharge. Three-month mortality was defined as death due to all causes within 3 months after the diagnosis of $\mathrm{PE}$.

\section{Statistical analysis}

Descriptive statistics are presented as medians $\left(25^{\text {th }}\right.$ to $75^{\text {th }}$ percentiles) or numbers (percentages). Fisher's exact test was used to analyze categorical data, and the MannWhitney U-test was used to compare continuous data. Logistic regression analyses using backward elimination were performed to identify the factors independently predicting RPVO occurrence. Independent variables and those with $\mathrm{P}$ values $<0.1$ in univariate analyses were included in multivariate analyses. The data are presented as adjusted odds ratios (ORs) with $95 \%$ confidence intervals (CIs). A twotailed $\mathrm{P}$ value $<0.05$ was considered to indicate significance. All statistical analyses were performed using SPSS software (version 21.0; IBM Corp., Armonk, NY, USA).

\section{Results}

\section{Clinical characteristics}

During the study period, 1,216 patients suffered from acute 


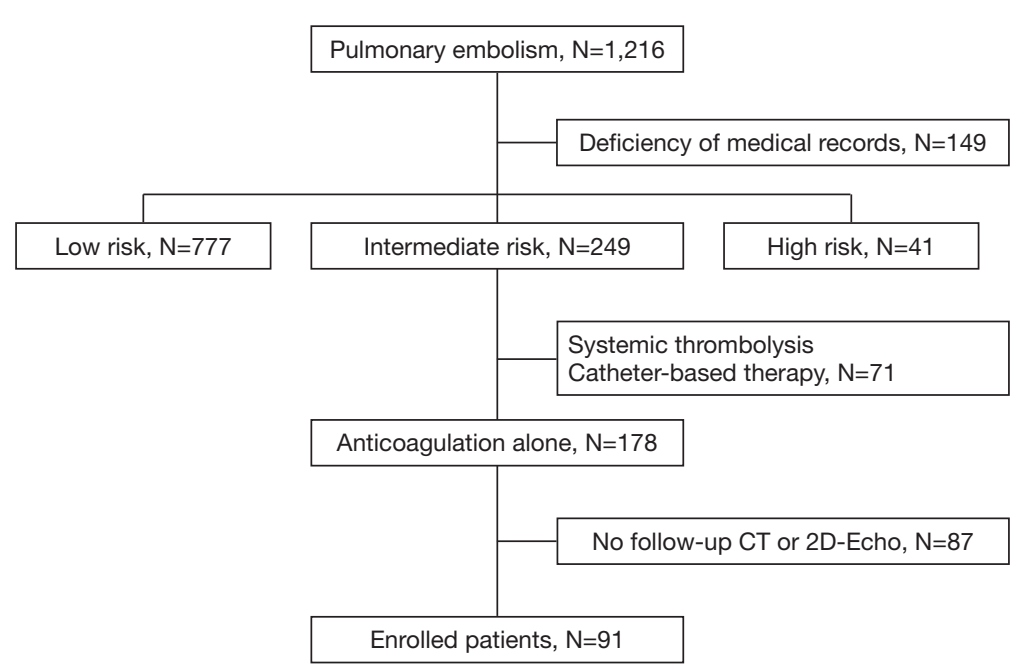

Figure 1 Flow chart of enrolled patients. CT, computed tomography; echo, echocardiography.

PE. From this group, risk stratification was not possible in 149 because of medical record deficiencies, and 818 were excluded because they were not considered to be at intermediate risk (low risk group, $\mathrm{n}=777$; high risk group, $\mathrm{n}=41$ ). Among the 249 patients with acute PE who were identified as being at intermediate risk, 71 had been treated with systemic thrombolysis or catheter-based therapy rather than anticoagulation monotherapy and 87 had no followup data. Thus, 91 patients were finally included in the study (Figure 1).

The clinical characteristics of the 91 patients are listed in Table 1. The median age was 72 years and 37 (40.7\%) were male. The body mass index, systolic BP, heart rate, and peripheral oxygen saturation at $\mathrm{PE}$ occurrence were $25 \mathrm{~kg} / \mathrm{m}^{2}, 120 \mathrm{mmHg}, 100$ beats/min, and $93 \%$, respectively. The most common comorbidities were solid cancer and chronic lung disease. PE in almost $20 \%$ of patients was due to conditions related to immobilization. The median pulmonary embolism severity index (PESI) was 99 points. Most pulmonary emboli in this study were central lesions (e.g., saddle-shaped or involving both main bronchi), and the median pulmonary artery pressure (PAP) at the occurrence of $\mathrm{PE}$ was $50 \mathrm{mmHg}$.

The management and prognosis of patients in this study are summarized in Table 2. Almost all of the patients were treated with heparin (e.g., conventional heparin or LMWH) as initial therapy and a NOAC and warfarin as maintenance therapy. All-cause mortality during the 3 months after the start of treatment was $5.5 \%$. The median follow-up period in included patients was 30 months. At the long-term follow-up examinations, 25 of 91 (27.5\%) patients were diagnosed with RPVO. Among the patients diagnosed with RPVO, 11 (44\%) suffered from persistent dyspnea.

\section{Comparison of baseline characteristics between patients with RPVO and those without RPVO}

We investigated the occurrence of RPVO during long-term follow-up to evaluate the appropriateness of anticoagulation monotherapy.

The baseline characteristics of the patients with and without RPVO are compared in Table 3. In those with RPVO, the median age was 71 years, and $48 \%$ were male. Patients in this group had a higher proportion of chronic lung disease than did patients without RPVO. The proportion of other diseases was similar in the two groups. Patients with RPVO had a lower proportion of provoked $\mathrm{PE}$ than those without RPVO, although the difference was not statistically significant. The severity of PE was similar in the two groups.

\section{Factors predicting the occurrence of RPVO}

Logistic regression was used to identify the factors associated with RPVO occurrence. According to the univariate analyses, chronic lung disease and a P/A ratio $>1.0$ documented on a chest $\mathrm{CT}$ scan obtained at presentation were significantly associated with the occurrence of RPVO. Multivariate logistic regression (using backward elimination) revealed chronic lung disease (OR, 
Table 1 Clinical characteristics of the patients in this study

\begin{tabular}{|c|c|}
\hline Variables & Patients $(n=91)$ \\
\hline Age (years)* & 72 [63-79] \\
\hline Male sex & $37(40.7)$ \\
\hline Body mass index $\left(\mathrm{kg} / \mathrm{m}^{2}\right)^{\star}$ & 25 [22-28] \\
\hline \multicolumn{2}{|c|}{ Initial clinical signs on presentation* } \\
\hline Systolic BP (mmHg) & 120 [110-139] \\
\hline Diastolic BP (mmHg) & 73 [70-80] \\
\hline Heart rate (beats/min) & $100[91-116]$ \\
\hline Respiratory rate (breaths/min) & 23 [20-26] \\
\hline $\mathrm{SpO}_{2}(\%)$ & 93 [88-95] \\
\hline \multicolumn{2}{|l|}{ Comorbidities } \\
\hline Chronic lung disease & $15(16.5)$ \\
\hline Chronic liver disease & $1(1.1)$ \\
\hline Congestive heart failure & $3(3.3)$ \\
\hline Chronic kidney disease & $3(3.3)$ \\
\hline Solid cancer & $21(23.1)$ \\
\hline Alleged thrombophilia & $4(4.4)$ \\
\hline \multicolumn{2}{|l|}{ Risk factors } \\
\hline Immobilization & $18(19.8)$ \\
\hline Recent surgery & $15(16.5)$ \\
\hline Previous DVT or PE & $12(13.2)$ \\
\hline Pregnancy & $2(2.2)$ \\
\hline Estrogen use & $1(1.1)$ \\
\hline Provoked PE & $46(50.5)$ \\
\hline PESI $^{*}$ & 99 [77-123] \\
\hline Central lesion in location of PE & $55(60.4)$ \\
\hline \multicolumn{2}{|l|}{ Chest CT findings on presentation } \\
\hline $\mathrm{RV} / \mathrm{LV}$ ratio $>0.9$ & $77(84.6)$ \\
\hline $\mathrm{P} / \mathrm{A}$ ratio $>1.0$ & $53(58.2)$ \\
\hline \multicolumn{2}{|l|}{ 2D-echo findings on presentation } \\
\hline D-shape & $44(48.4)$ \\
\hline RV dilatation & $63(69.2)$ \\
\hline RV hypokinesia & $66(72.5)$ \\
\hline Systolic PAP $(\mathrm{mmHg})^{*}$ & 50 [43-62] \\
\hline
\end{tabular}

*, data are presented as median $\left[25^{\text {th }}-75^{\text {th }}\right.$ percentiles]. Other variables are presented as number (percent). BP, blood pressure; $\mathrm{SpO}_{2}$, peripheral oxygen saturation; DVT, deep vein thrombosis; $\mathrm{PE}$, pulmonary embolism; PESI, pulmonary embolism severity index; CT, computed tomography; RV/LV ratio, right ventricle diameter/left ventricle diameter ratio; P/A ratio, main pulmonary artery diameter/ascending aorta diameter ratio; echo, echocardiography; PAP, pulmonary artery pressure.
Table 2 The management and prognosis of patients in this study

\begin{tabular}{lc}
\hline Variables & Patients $(\mathrm{n}=91)$ \\
\hline Anticoagulants within 1 week & $34(37.4)$ \\
Conventional heparin & $54(59.3)$ \\
LMWH & $3(3.3)$ \\
NOAC & \\
Anticoagulation switch for maintenance & $44(48.4)$ \\
Warfarin & $3(3.3)$ \\
LMWH & $44(48.4)$ \\
NOAC & \\
Adverse events & $0(0.0)$ \\
Major bleeding & $15(16.5)$ \\
Minor bleeding & \\
All-cause mortality & $0(0.0)$ \\
In-hospital mortality & $5(5.5)$ \\
3-month mortality & $25(27.5)$ \\
\hline RPO &
\end{tabular}

Data are presented as number (percent). LMWH, Iowmolecular weight heparin; NOAC, non-vitamin $\mathrm{K}$ antagonist oral anticoagulant; RPVO, residual pulmonary vascular obstruction.

4.14; $95 \% \mathrm{CI}, 1.243-13.797 ; \mathrm{P}=0.021)$, and a $\mathrm{P} / \mathrm{A}$ ratio $>1.0$ documented on a chest $\mathrm{CT}$ scan at presentation (OR, 3.46; 95\% CI, 1.113-10.770; $\mathrm{P}=0.032$ ) were significant independent predictors of RPVO occurrence (Table 4).

The incidence of RPVO in patients without these two factors was only $9.7 \%$, but in those with the two factors it was $60 \%$ (Figure 2; $\mathrm{P}=0.004$ ). In the latter group, the sensitivity, specificity, positive predictive value (PPV), negative predictive value (NPV), and accuracy of the two factors in predicting RPVO occurrence were $66.7 \%, 87.5 \%$, $60 \%, 90.3 \%$, and $82.9 \%$, respectively (Table S1).

\section{Discussion}

This study investigated whether anticoagulation monotherapy was appropriate in patients with intermediate risk $\mathrm{PE}$, and the factors that independently predicted RPVO. Our results showed that almost $30 \%$ of patients undergoing anticoagulation monotherapy showed RPVO on follow-up imaging. The occurrence of RPVO was higher among patients with underlying chronic lung disease and a P/A ratio $>1$ on the chest $\mathrm{CT}$ scan obtained 
Table 3 Comparison of baseline characteristics between patients with RPVO and those without RPVO

\begin{tabular}{|c|c|c|c|}
\hline Variables & \multicolumn{2}{|c|}{ RPVO } & $P$ value \\
\hline Age (years) ${ }^{*}$ & $71[54-76]$ & 74 [64-80] & 0.227 \\
\hline Male sex & $12(48.0)$ & $25(37.9)$ & 0.475 \\
\hline Body mass index $\left(\mathrm{kg} / \mathrm{m}^{2}\right)^{\star}$ & 26 [23-28] & 24 [22-28] & 0.197 \\
\hline Systolic BP (mmHg) & $120[110-138]$ & $120[110-140]$ & 0.881 \\
\hline Diastolic BP (mmHg) & $73[71-80]$ & 72 [69-83] & 0.675 \\
\hline Heart rate (beats/min) & 98 [89-115] & $101[91-117]$ & 0.576 \\
\hline Respiratory rate (breaths/min) & 23 [20-26] & 23 [20-27] & 0.906 \\
\hline Chronic liver disease & $0(0.0)$ & $1(1.5)$ & 1.000 \\
\hline Congestive heart failure & $1(4.0)$ & $2(3.0)$ & 1.000 \\
\hline Chronic kidney disease & $0(0.0)$ & $3(4.5)$ & 0.559 \\
\hline Active cancer & $7(28.0)$ & $14(21.2)$ & 0.579 \\
\hline Alleged thrombophilia & $1(4.0)$ & $3(4.5)$ & 1.000 \\
\hline \multicolumn{4}{|l|}{ Risk factors } \\
\hline Immobilization & $2(8.0)$ & $16(24.2)$ & 0.138 \\
\hline PESI $^{*}$ & 84 [74-112] & $100[79-125]$ & 0.382 \\
\hline Central lesion in location of PE & $16(64.0)$ & $39(59.1)$ & 0.602 \\
\hline \multicolumn{4}{|l|}{ Chest CT findings on presentation } \\
\hline $\mathrm{RV} / \mathrm{LV}$ ratio $>0.9$ & $23(92.0)$ & $54(81.8)$ & 0.503 \\
\hline $\mathrm{P} / \mathrm{A}$ ratio $>1.0$ & $19(76.0)$ & $34(51.5)$ & 0.029 \\
\hline \multicolumn{4}{|l|}{ 2D-echo findings on presentation } \\
\hline D-shape & $14(56.0)$ & $30(45.5)$ & 0.473 \\
\hline RV dilatation & $18(72.0)$ & $45(68.2)$ & 0.793 \\
\hline RV hypokinesia & $17(68.0)$ & $49(74.2)$ & 0.786 \\
\hline Systolic PAP $(\mathrm{mmHg})^{*}$ & $56[45-71]$ & $48[41-60]$ & 0.075 \\
\hline
\end{tabular}

${ }^{*}$, data are presented as median $\left[25^{\text {th }}-75^{\text {th }}\right.$ percentiles]. Other variables are presented as number (percent). RPVO, residual pulmonary vascular obstruction; $\mathrm{BP}$, blood pressure; $\mathrm{SpO}_{2}$, peripheral oxygen saturation; DVT, deep vein thrombosis; PE, pulmonary embolism; PESI, pulmonary embolism severity index; CT, computed tomography; RV/LV ratio, right ventricle diameter/left ventricle diameter ratio; P/A ratio, main pulmonary artery diameter/ascending aorta diameter ratio; echo, echocardiography; PAP, pulmonary artery pressure. 
Table 4 Factors predicting the occurrence of RPVO

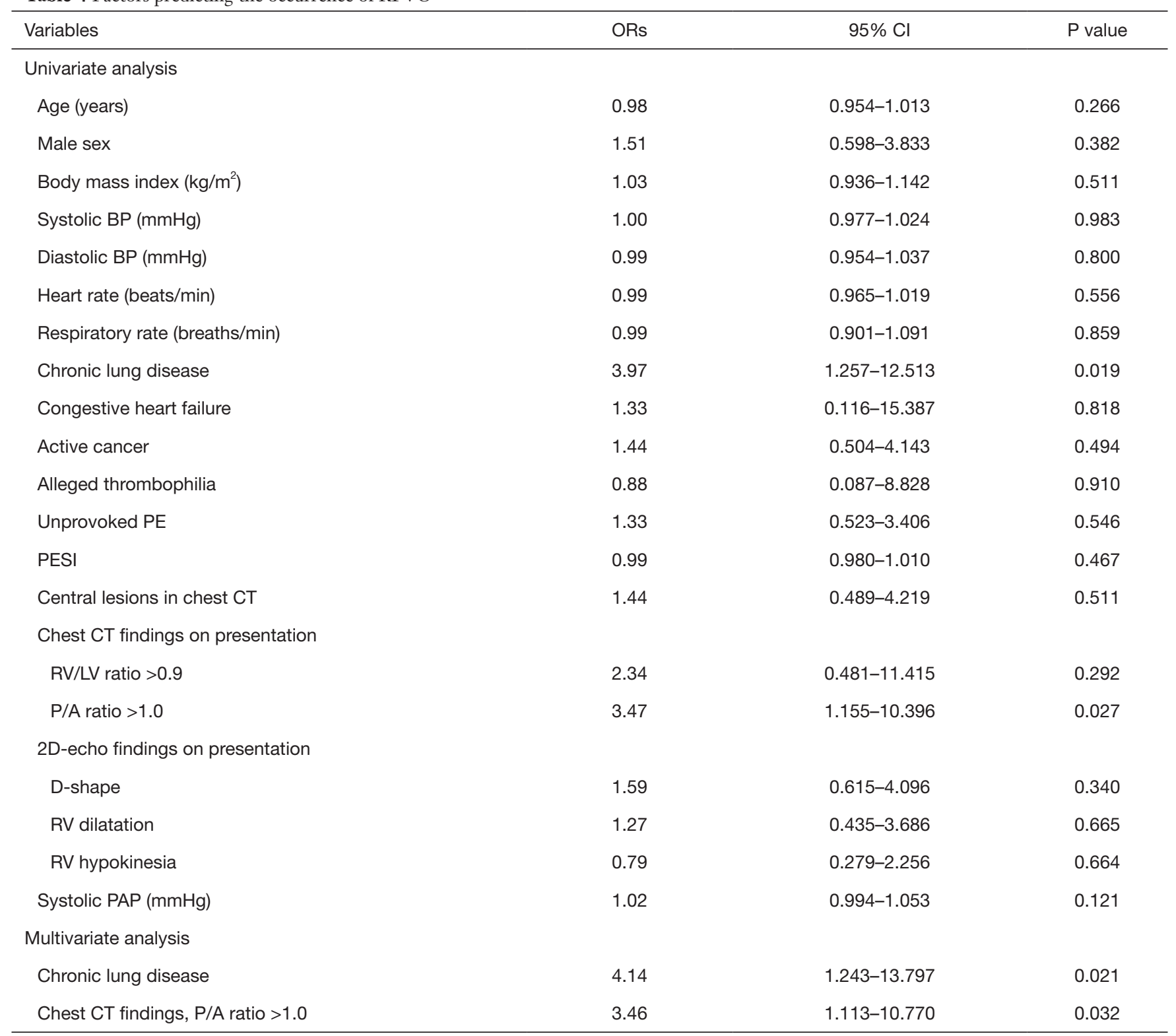

Multivariate logistic regression analysis that used backward elimination was performed to predict RPVO after adjusting for two variables (the presence of chronic lung disease, chest CT findings, P/A ratio >1.0). RPVO, residual pulmonary vascular obstruction; OR, odds ratio; Cl, confidence interval; BP, blood pressure; PE, pulmonary embolism; PESI, pulmonary embolism severity index; CT, computed tomography; RV/LV ratio, right ventricle diameter/left ventricle diameter ratio; P/A ratio, main pulmonary artery diameter/ascending aorta diameter ratio; echo, echocardiography; PAP, pulmonary artery pressure.

at initial presentation than in patients without these factors. Therefore, clinicians should pay close attention to differentiate between persistent dyspnea due to RPVO and dyspnea due to underlying disease in patients with chronic lung disease, particularly those with a P/A ratio $>1$ on the initial chest CT scan. If patients have unexplained dyspnea,
CTEPD should be considered and CTEPD patients should be treated to improve quality of life.

Most studies on PE aimed to define an appropriate treatment for reducing mortality. Patients with PE were treated according to risk stratification based on the results of these studies (1-7). In patients at intermediate risk, PE 


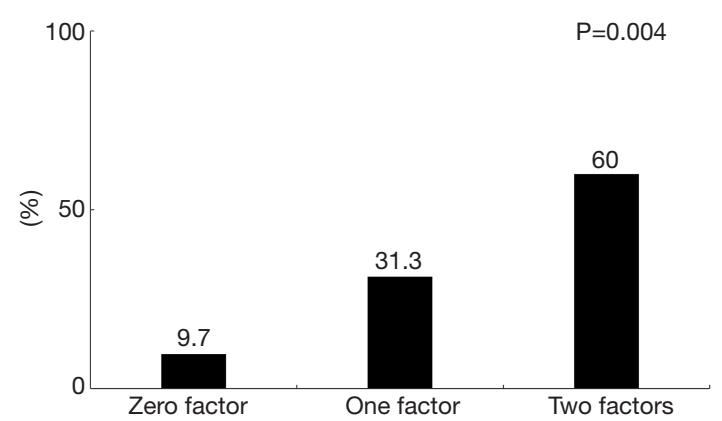

Figure 2 The incidence of RPVO based on the number of risk factors. RPVO, residual pulmonary vascular obstruction.

treatment was also decided based on the evaluation of bleeding risk and the likely benefit in terms of reducing mortality $(1-7,11)$. As treatment for acute PE was improved, mortality due to acute PE has decreased remarkably (1-7). In our study, hospital mortality following acute PE was $0 \%$ and 3 -month mortality was $5.5 \%$, with the latter including the progression of comorbidities, such as cancer. Anticoagulation monotherapy in patients with intermediate risk $\mathrm{PE}$ is effective for reducing mortality when considering the risk-benefit ratio, but anticoagulation monotherapy did not seem to be a sufficient treatment to reduce RPVO because almost $30 \%$ of patients with anticoagulation monotherapy showed RPVO on followup imaging. However, this result was similar to that of patients in our initial cohort who were first treated with systemic thrombolysis or catheter-based therapy instead of anticoagulation monotherapy $(27.5 \%$ vs. $31.1 \% ; \mathrm{P}=0.690$, Figure S1). Therefore, the type of initial treatment (anticoagulation alone $v s$. systemic thrombolysis or catheter-based therapy) did not affect the occurrence of RPVO, similar to previous studies $(24,25)$. Also, in our study, $44 \%$ of patients with RPVO (11 of 25 patients) suffered from persistent dyspnea. Because almost $50 \%$ of intermediate-risk PE patients with RPVO suffered from persistent dyspnea, clinicians should pay close attention to the presence of CTEPD in these patients.

International guidelines only recommend followup imaging in patients with persistent symptoms due to $\mathrm{PE}$. However, in clinical practice, it is difficult to differentiate between dyspnea due to PE and dyspnea due to comorbidities, particularly in elderly patients. To solve this problem, several examinations, such as cardiopulmonary exercise testing (CPET) and exercise right heart catheterization (ex-RHC), may be needed $(1,2,14,26)$.
Because clinicians cannot apply these examinations in all intermediate-risk PE patients, selecting patients at risk of RPVO is important. Previous studies have proposed a predictive model for CTEPH, we could not easily apply to intermediate-risk PE patients because of many variables in this model $(14,18,27,28)$. In our study, patients with underlying chronic lung disease and a P/A ratio $>1$ determined from the chest CT scan obtained at the initial presentation were at higher risk of RPVO than patients without these factors. These variables can be used to evaluate patients with RPVO, even by non-experts. Because we can easily predict RPVO on the day of admission, patients with risk factors for RPVO could be more carefully evaluated for persistent dyspnea through history-taking, CPET, and ex-RHC.

A mechanism explaining the associations of risk factors with RPVO has been suggested: chronic lung diseases are associated with systemic inflammation and the release of systemic inflammatory factors, such as C-reactive protein, fibrinogen, and tumor necrosis factor- $\alpha$, which contribute to thrombotic events. In addition, PH induced by chronic lung disease can impede the pulmonary circulation (29-33). A P/ A ratio $>1$ on the chest $\mathrm{CT}$ scan is an indicator of persistent $\mathrm{PH}$, especially in patients with chronic lung disease $(34,35)$. The $\mathrm{RV} / \mathrm{LV}$ ratio $>0.9$, interventricular septum flattening and paradoxical septal bowing on chest CT are an indicator of $\mathrm{RV}$ dysfunction, but these findings were not associated with RPVO in this study. Considering a P/A ratio $>1$ on the chest $\mathrm{CT}$ is an indicator of persistent $\mathrm{PH}$, persistent $\mathrm{PH}$ may be a better predictor of RPVO than transient RV dysfunction.

This study had several limitations. First, the total number of enrolled patients was relatively small compared with the number of screened patients, because international guidelines do not recommend routine follow-up imaging in patients with PE. Moreover, the concept of CTEPD was not well-defined between 2012 and 2017. However, the number of patients in our study was sufficient to reveal an association between anticoagulation monotherapy in patients with intermediate risk $\mathrm{PE}$ and the occurrence of RPVO. Second, patients with PE or RPVO were diagnosed by chest $C T$. Although ventilation-perfusion scintigraphy is the most effective modality to diagnose PE and RPVO, chest $\mathrm{CT}$ is easier to perform because of the relatively low cost in the Republic of Korea $(<\$ 200)$. In addition, given that high-quality CT angiography is adequate for diagnosing proximal CTEPD (sensitivity $=99 \%$, specificity $=97 \%$ ), chest $\mathrm{CT}$ may also be valuable in the treatment of the disease $(1,2,14)$. 
In conclusion, anticoagulation monotherapy did not seem to be a sufficient treatment to reduce RPVO, but the outcome was similar to that of patients treated with other therapies. Therefore, considering the risk-benefit ratio, we do not need to change the initial treatment as systemic thrombolytic therapy or catheter-based therapy in patient with intermediate risk PE. Underlying chronic lung disease and a $\mathrm{P} / \mathrm{A}$ ratio $>1$ on the initial chest $\mathrm{CT}$ scan predicted the occurrence of RPVO. Therefore, we should carefully assess persistent of dyspnea and exercise limitations using various methods in patients with these risk factors, to detect the occurrence of CTEPD earlier. Additional studies with larger patient cohorts are warranted to validate our findings.

\section{Acknowledgments}

Funding: None.

\section{Footnote}

Reporting Checklist: The authors have completed the STROBE reporting checklist. Available at https://dx.doi. org/10.21037/jtd-21-403

Data Sharing Statement: Available at https://dx.doi. org/10.21037/jtd-21-403

Peer Review File: Available at https://dx.doi.org/10.21037/ jtd-21-403

Conflicts of Interest: All authors have completed the ICMJE uniform disclosure form (available at https://dx.doi. org/10.21037/jtd-21-403). The authors have no conflicts of interest to declare.

Ethical Statement: The authors are accountable for all aspects of the work in ensuring that questions related to the accuracy or integrity of any part of the work are appropriately investigated and resolved. The study was conducted in accordance with the Declaration of Helsinki (as revised in 2013). The study was approved by the Institutional Review Boards (IRBs) of all participating hospitals and the Korea Medical Center IRB (approval No.: 2020GR0057). We ensured protection of patient privacy and anonymity. The need for informed consent was waived because of the retrospective nature of the study.

Open Access Statement: This is an Open Access article distributed in accordance with the Creative Commons Attribution-NonCommercial-NoDerivs 4.0 International License (CC BY-NC-ND 4.0), which permits the noncommercial replication and distribution of the article with the strict proviso that no changes or edits are made and the original work is properly cited (including links to both the formal publication through the relevant DOI and the license). See: https://creativecommons.org/licenses/by-nc-nd/4.0/.

\section{References}

1. Konstantinides SV, Meyer G, Becattini C, et al. 2019 ESC Guidelines for the diagnosis and management of acute pulmonary embolism developed in collaboration with the European Respiratory Society (ERS): The Task Force for the diagnosis and management of acute pulmonary embolism of the European Society of Cardiology (ESC). Eur Respir J 2019;54:1901647.

2. Konstantinides SV, Meyer G, Becattini C, et al. 2019 ESC Guidelines for the diagnosis and management of acute pulmonary embolism developed in collaboration with the European Respiratory Society (ERS). Eur Heart J 2020;41:543-603.

3. Di Nisio M, van Es N, Büller HR, et al. Deep vein thrombosis and pulmonary embolism. Lancet 2016;388:3060-73.

4. Barrios D, Yusen RD, Jiménez D, et al. Risk stratification for proven acute pulmonary embolism: what information is needed? Semin Respir Crit Care Med 2017;38:11-7.

5. Becattini C, Agnelli G, Lankeit M, et al. Acute pulmonary embolism: mortality prediction by the 2014 European Society of Cardiology risk stratification model. Eur Respir J 2016;48:780-6.

6. Agnelli G, Becattini C, et al. Anticoagulant treatment for acute pulmonary embolism: a pathophysiology-based clinical approach. Eur Respir J 2015;45:1142-9.

7. Kearon C, Akl EA, Ornelas J, et al. Antithrombotic therapy for VTE disease: CHEST guideline and expert panel report. Chest 2016;149:315-52.

8. Meyer G, Vicaut E, Danays T, et al. Fibrinolysis for patients with intermediate-risk pulmonary embolism. N Engl J Med 2014;370:1402-11.

9. Furfaro D, Stephens RS, Streiff MB, et al. Catheterdirected Thrombolysis for Intermediate-Risk Pulmonary Embolism. Ann Am Thorac Soc 2018;15:134-44.

10. Avgerinos ED, Saadeddin Z, Abou Ali AN, et al. A metaanalysis of outcomes of catheter-directed thrombolysis for high- and intermediate-risk pulmonary embolism. J Vasc 
Surg Venous Lymphat Disord 2018;6:530-40.

11. Rali PM, Criner GJ, et al. Submassive pulmonary embolism. Am J Respir Crit Care Med 2018;198:588-98.

12. Dzikowska-Diduch O, Kostrubiec M, Kurnicka K, et al. "The post-pulmonary syndrome - results of echocardiographic driven follow up after acute pulmonary embolism". Thromb Res 2020;186:30-5.

13. Bonnefoy PB, Margelidon-Cozzolino V, Catella-Chatron J, et al. What's next after the clot? Residual pulmonary vascular obstruction after pulmonary embolism: From imaging finding to clinical consequences. Thromb Res 2019;184:67-76.

14. Delcroix M, Torbicki A, Gopalan D, et al. ERS statement on chronic thromboembolic pulmonary hypertension. Eur Respir J 2021;57:2002828.

15. Klok FA, van der Hulle T, den Exter PL, et al. The postPE syndrome: a new concept for chronic complications of pulmonary embolism. Blood Rev 2014;28:221-6.

16. Wang J, Xu M, Sun N, et al. Factors associating with the presence of residual thrombosis after 3-month treatment of acute pulmonary embolism. J Thromb Thrombolysis 2018;45:27-35.

17. Robin P, Eddy M, Sikora L, et al. Residual pulmonary vascular obstruction and recurrence after acute pulmonary embolism: protocol for a systematic review and metaanalysis of individual participant data. BMJ Open 2018;8:e023939.

18. Klok FA, Dzikowska-Diduch O, Kostrubiec M, et al. Derivation of a clinical prediction score for chronic thromboembolic pulmonary hypertension after acute pulmonary embolism. J Thromb Haemost 2016;14:121-8.

19. Kahn SR, Hirsch AM, Akaberi A, et al. Functional and exercise limitations after a first episode of pulmonary embolism: results of the ELOPE prospective cohort study. Chest 2017;151:1058-68.

20. Held M, Kolb P, Grün M, et al. Functional characterization of patients with chronic thromboembolic disease. Respiration 2016;91:503-9.

21. Inami T, Kataoka M, Kikuchi H, et al. Balloon pulmonary angioplasty for symptomatic chronic thromboembolic disease without pulmonary hypertension at rest. Int J Cardiol 2019;289:116-8.

22. Olgun Yıldızeli Ş, Kepez A, Taş S, et al. Pulmonary endarterectomy for patients with chronic thromboembolic disease. Anatol J Cardiol 2018;19:273-8.
23. Wiedenroth CB, Olsson KM, Guth S, et al. Balloon pulmonary angioplasty for inoperable patients with chronic thromboembolic disease. Pulm Circ 2018;8:2045893217753122.

24. Barco S, Russo M, Vicaut E, et al. Incomplete echocardiographic recovery at 6 months predicts longterm sequelae after intermediate-risk pulmonary embolism. A post-hoc analysis of the Pulmonary Embolism Thrombolysis (PEITHO) trial. Clin Res Cardiol 2019;108:772-8.

25. Konstantinides SV, Vicaut E, Danays T, et al. Impact of thrombolytic therapy on the long-term outcome of intermediate-risk pulmonary embolism. J Am Coll Cardiol 2017;69:1536-44.

26. Klok FA, Couturaud F, Delcroix M, et al. Diagnosis of chronic thromboembolic pulmonary hypertension after acute pulmonary embolism. Eur Respir J 2020;5 5:2000189.

27. Kim NH, Delcroix M, Jais X, et al. Chronic thromboembolic pulmonary hypertension. Eur Respir J 2019;53:1801915.

28. Guérin L, Couturaud F, Parent F, et al. Prevalence of chronic thromboembolic pulmonary hypertension after acute pulmonary embolism. Prevalence of CTEPH after pulmonary embolism. Thromb Haemost 2014;112:598-605.

29. Aleva FE, Voets LWLM, Simons SO, et al. Prevalence and localization of pulmonary embolism in unexplained acute exacerbations of COPD: a systematic review and metaanalysis. Chest 2017;151:544-54.

30. Park SH. Pulmonary embolism is more prevalent than deep vein thrombosis in cases of chronic obstructive pulmonary disease and interstitial lung diseases. Springerplus 2016;5:1777.

31. Saghazadeh A, Rezaei N, et al. Inflammation as a cause of venous thromboembolism. Crit Rev Oncol Hematol 2016;99:272-85.

32. Esmon CT. The interactions between inflammation and coagulation. Br J Haematol 2005;131:417-30.

33. Seeger W, Adir Y, Barberà JA, et al. Pulmonary hypertension in chronic lung diseases. J Am Coll Cardiol 2013;62:D109-16.

34. Ortaç Ersoy E, Durusu Tanriover M, Öcal S, et al. Measurement of pulmonary artery to aorta ratio in computed tomography is correlated with pulmonary artery pressure in critically ill chronic obstructive pulmonary 
disease patients. J Crit Care 2016;33:42-6.

35. Coste F, Benlala I, Dournes G, et al. Assessing pulmonary hypertension in COPD. Is there a role for computed

Cite this article as: Chang Y, Moon JY, Park JH, Choi S, Lee HP, Sim JK, Lee YS. Predictors of residual pulmonary vascular obstruction after anticoagulation monotherapy in patients with intermediate-risk pulmonary embolism. J Thorac Dis 2021;13(7):4217-4227. doi: 10.21037/jtd-21-403 tomography? Int J Chron Obstruct Pulmon Dis 2019;14:2065-79. 
Supplementary

Table S1 Performance for predicting the occurrence of RPVO in patients with chronic lung disease and P/A ratio >1.0 in chest CT

\begin{tabular}{lccccc}
\hline & Sensitivity & Specificity & PPV & NPV & Accuracy \\
\hline Patients with two factors & $66.7 \%$ & $87.5 \%$ & $60 \%$ & $90.3 \%$ & $82.9 \%$ \\
\hline
\end{tabular}

RPVO, residual pulmonary vascular obstruction; P/A ratio, the ratio of the diameters of the main pulmonary artery and ascending aorta; $\mathrm{CT}$, computed tomography; PPV, positive predictive value; NPV, negative predictive value.

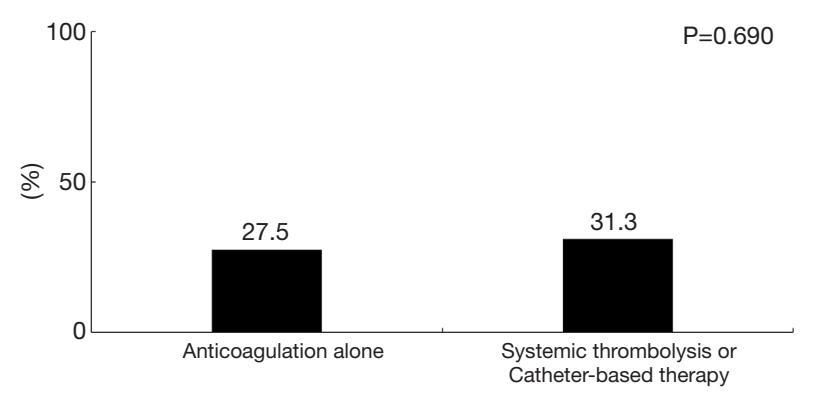

Figure S1 The incidence of RPVO based on treatments. RPVO, residual pulmonary vascular obstruction. 